\title{
The Medial Prefrontal and Orbitofrontal Cortices Differentially Regulate Dopamine System Function
}

\author{
Daniel J Lodge*,I \\ 'Department of Pharmacology and Center for Biomedical Neuroscience, University of Texas Health Science Center, San Antonio, TX, USA
}

\begin{abstract}
The prefrontal cortex (PFC) is essential for top-down control over higher-order executive function. In this study we demonstrate that the medial prefrontal cortex (MPFC) and orbitofrontal cortex (OFC) differentially regulate VTA dopamine neuron activity, and furthermore, the pattern of activity in the PFC drastically alters the dopamine neuron response. Thus, although single-pulse activation of the MPFC either excites or inhibits equivalent numbers of dopamine neurons, activation of the OFC induces a primarily inhibitory response. Moreover, activation of the PFC with a pattern that mimics spontaneous burst firing of pyramidal neurons produces a strikingly different response. Specifically, burst-like activation of the mPFC induces a massive increase in dopamine neuron firing, whereas a similar pattern of OFC activation largely inhibits dopamine activity. Taken together, these data demonstrate that the mPFC and OFC differentially regulate dopamine neuron activity, and that the pattern of cortical activation is critical for determining dopamine system output.
\end{abstract}

Neuropsychopharmacology (2011) 36, 1227-1236; doi:10.1038/npp.2011.7; published online 9 February 20II

Keywords: prefrontal cortex; orbitofrontal cortex; dopamine; VTA

\section{INTRODUCTION}

The prefrontal cortex (PFC) is essential for top-down control over higher-order executive function. The two major prefrontal regions implicated in the regulation of dopamine system function are the medial prefrontal (mPFC) and orbitofrontal (OFC) cortices, regions that carry out independent, but complementary forms of cognitive processing in changing environmental conditions (Floresco et al, 2006; McAlonan and Brown, 2003; O'Doherty et al, 2003). There is a significant literature demonstrating that the MPFC regulates dopamine neuron activity (Aston-Jones et al, 2009; Overton et al, 1996; Tong et al, 1996, 1998). Electrical stimulation of the mPFC induces both inhibitory and excitatory responses in dopamine neurons (Aston-Jones et al, 2009; Overton et al, 1996; Tong et al, 1996, 1998). This likely reflects the specific anatomy of mPFC afferents to the VTA. Thus, mPFC afferents target only those dopamine neurons that project back to the PFC (Carr and Sesack, 2000). In addition, the mPFC innervates GABAergic neurons within the VTA that project to the NAc as well as providing local axonal collaterals within the VTA (Carr and

*Correspondence: Dr DJ Lodge, UTHSCSA, Mail Code 7764, 7703 Floyd Curl Drive, San Antonio, TX 78229-3900, USA, Tel: + I 210567 4188, Fax: + I 210567 4303, E-mail: LodgeD@UTHSCSA.edu

Received I4 September 2010; revised 6 January 2011; accepted II January 2011
Sesack, 2000; Sesack and Carr, 2002). Thus, excitatory responses observed in dopamine neurons following mPFC stimulation are likely limited to mesocortical dopamine neurons, whereas inhibitory responses may occur secondary to activation of GABAergic neurons.

In contrast to the literature examining the function of the mPFC, a role for the OFC in the regulation of dopamine system function has not yet been demonstrated but is of critical importance, particularly given recent preclinical studies by Schoenbaum et al (2009) demonstrating the signaling of reward expectation by OFC neurons (Takahashi et al, 2009). Specifically, OFC neurons demonstrate a greater increase in activity before predictable rewards when compared with activity preceding a delayed, unpredictable reward (Takahashi et al, 2009). This is essentially the opposite of what is observed in dopamine neurons, that is, an increased activity to an unexpected reward, suggesting that the OFC may negatively regulate dopamine neuron activity. Indeed, the data presented in this study demonstrate that OFC activation results in a strong inhibition of spontaneous activity within the VTA.

It is important to note that neuronal activity within the PFC is not limited to single-spike firing. Thus, pyramidal neurons of the cortex display transient elevations in activity in response to cognitive tasks (Homayoun and Moghaddam, 2006a; Jackson et al, 2004; Jung et al, 1998). Furthermore, a proportion of pyramidal neurons, particularly those located in the deeper parts of layer 5 (Chagnac-Amitai et al, 1990), 
have also been demonstrated to fire high-frequency bursts of action potentials during task performance (BurgosRobles et al, 2007; Chang et al, 2010; Laviolette et al, 2005; Santini and Porter, 2010; Wang and Goldman-Rakic, 2004; Zhang and Shi, 1999). This distinct firing pattern results in large changes in neurotransmitter release, as well as the liberation of peptide neuromodulators (Whim and Lloyd, 1989) that are suggested to enhance the signaling of salient events (Cooper, 2002; Lisman, 1997; Miller and Cohen, 2001). Thus, understanding how burst-like activation of the PFC regulates dopamine system function is critical to understanding the consequence of this firing pattern with regard to information processing of salient events. Indeed, the data presented in this study demonstrate that the pattern of cortical activation is critical for determining dopamine system output.

\section{MATERIALS AND METHODS}

All experiments were performed in accordance with the guidelines outlined in the USPHS Guide for the Care and Use of Laboratory Animals and were approved by the institutional animal care and use committee of the University of Texas Health Science Center, San Antonio.

\section{Dopamine Neuron Electrophysiology}

Male Sprague Dawley rats (250-350 g) were anaesthetized with chloral hydrate $(400 \mathrm{mg} / \mathrm{kg}$ i.p.) and placed in a stereotaxic apparatus. Chloral hydrate was utilized for all recordings as under this anesthetic, the evoked cortical activity is relatively unaltered (Angel and Gratton, 1982) and dopamine neuron activity states more closely resemble that observed in freely moving rats (Hyland et al, 2002). Anesthesia was maintained by supplemental administration of chloral hydrate as required to maintain suppression of limb compression withdrawal reflex. A core body temperature of $37^{\circ} \mathrm{C}$ was sustained by a thermostatically controlled heating pad (TCAT-2LV; KOPF). Bipolar concentric stimulating electrodes (KOPF/Rhodes NEX100) were implanted in the $\mathrm{mPFC}(\mathrm{A} / \mathrm{P}+3.0, \mathrm{M} / \mathrm{L}+0.5, \mathrm{D} / \mathrm{V}-5.0 \mathrm{~mm}$ from bregma $)$ and OFC $(\mathrm{A} / \mathrm{P}+3.0, \mathrm{M} / \mathrm{L}+3.5, \mathrm{D} / \mathrm{V}-5.0 \mathrm{~mm}$ from bregma). Glass extracellular microelectrodes (impedance 6-14 M $\Omega$ ) were lowered into the VTA (A/P -5.3, M/L $+0.6 \mathrm{~mm}$ from bregma and -6.5 to $-9.0 \mathrm{~mm}$ ventral of brain surface) using a hydraulic microdrive (model 640; KOPF) and the activity of the population of dopamine neurons was determined by counting the number of spontaneously active dopamine neurons encountered while making 6-9 vertical passes, separated by $200 \mu \mathrm{m}$, in a predetermined pattern to sample equivalent regions of the VTA. Spontaneously active dopamine neurons were identified with open filter settings (low cutoff: $30 \mathrm{~Hz}$, high cutoff: $30 \mathrm{kHz}$ ) using previously established electrophysiological criteria (Grace and Bunney, 1983), including an action potential $>2 \mathrm{~ms}$ and a firing rate from 0.5 to $12 \mathrm{~Hz}$. It should be noted that dopamine neuron waveforms identified under different filter conditions (Ungless et al, 2004), or from immature rats in vitro (Margolis et al, 2006), may not necessarily reflect those obtained with open filter settings in adult rats (Grace et al, 2007).
Once a stable dopamine neuron was identified (signal-tonoise typically greater than $3: 1$ ), low-cutoff filter settings were increased (to 100 or $300 \mathrm{~Hz}$ ) to minimize stimulus artifacts and single-current pulses $(0.25 \mathrm{~ms} ; 1.0 \mathrm{~mA})$ delivered at a rate of $0.5 \mathrm{~Hz}$ to the $\mathrm{mPFC}$ for $1 \mathrm{~min}$ followed by activation of the OFC $(0.25 \mathrm{~ms} ; 1.0 \mathrm{~mA} ; 0.5 \mathrm{~Hz})$ for an additional $1 \mathrm{~min}$. Trains of stimuli (four pulses; $65 \mathrm{~Hz}$, $1 \mathrm{~mA}, 0.25 \mathrm{~ms}$ ), used to mimic cortical burst firing (Laviolette et al, 2005), were then delivered at a rate of $0.5 \mathrm{~Hz}$ to $\mathrm{mPFC}$ for $1 \mathrm{~min}$ and the OFC for an additional $1 \mathrm{~min}$. A subset of neurons ( $\sim 40 \%)$ were recorded with the OFC stimulation performed first. To determine whether a dopamine neuron was excited or inhibited by the electrical stimulation, smoothed (second-order polynomial of three neighbors) peristimulus time histograms were generated and a criteria of $\geqslant 2$ consecutive bins $(50 \mathrm{~ms})$ that were greater (for excited neurons) or lower (for inhibited neurons) than 2 SD from the average baseline was utilized (Aston-Jones and Bloom, 1981; Clayton et al, 2004; Nishiike et al, 1997; Steinfels et al, 1983).

\section{Drug Administration}

For acute administration of drugs into the PFC, rats were implanted with a 23 -gauge cannula $2.0 \mathrm{~mm}$ dorsal to the $\mathrm{mPFC}(\mathrm{A} / \mathrm{P}+3.0, \mathrm{M} / \mathrm{L}+0.5, \mathrm{D} / \mathrm{V}-3.0 \mathrm{~mm}$ from bregma $)$ or OFC (A/P +3.0, M/L + 3.5, D/V $-3.0 \mathrm{~mm}$ from bregma). Chemical activation or disinhibition of the PFC was performed with $N$-methyl-D-aspartic acid (NMDA: $0.75 \mu \mathrm{g}$ per $0.5 \mu \mathrm{l}$ in Dulbecco's PBS) or $1(\mathrm{~S}), 9(\mathrm{R})-(-)$-bicuculline methbromide $(0.1 \mu \mathrm{g}$ per $0.5 \mu \mathrm{l}$ in Dulbecco's $\mathrm{PBS})$, respectively, infused through a 30-gauge injection cannula protruding $2.0 \mathrm{~mm}$ past the end of the implanted guide cannula. The injection cannula was left in situ for the duration of the experiments to ensure adequate diffusion of drug into the surrounding tissue. Rats received only one injection in either mPFC or OFC and dopamine neuron activity was typically recorded from $10 \mathrm{~min}$ to $2 \mathrm{~h}$ following infusions. Three parameters of activity were measured: (1) population activity (defined as the number of spontaneously active dopamine neurons recorded per electrode track), (2) basal firing rate, and (3) the proportion of action potentials occurring in bursts (defined as the occurrence of two spikes with an interspike interval of $<80 \mathrm{~ms}$, and the termination of the burst defined as the occurrence of an interspike interval of $>160 \mathrm{~ms}$; Grace and Bunney, 1984a). For NMDA experiments, data were compared with vehicletreated rats $(0.5 \mu \mathrm{l}$ Dulbecco's PBS into either the OFC or $\mathrm{mPFC}$ ), whereas within-subject comparisons were performed in bicuculline-treated rats, once drug effects had subsided.

NMDA and bicuculline were injected at doses previously demonstrated to augment afferent input to the mesolimbic dopamine system for electrophysiological studies (Floresco et al, 2003; Lodge and Grace, 2006a,b). Furthermore, it has been demonstrated previously that NMDA augments singleunit pyramidal cell activity (Lodge and Grace, 2006a) and that the enhanced activity persists for $>2 \mathrm{~h}$ (Lodge and Grace, 2006a, b). In contrast, bicuculline induces spike-wave discharges that have been demonstrated to correspond with coordinated bursting of pyramidal neurons (Steriade et al, 1998). To examine the duration of the effects of bicuculline, 
local field potentials were examined during drug administration, in a separate group of rats. Thus, guide cannula were implanted into the PFC with a $20^{\circ}$ angle to the midline $2.0 \mathrm{~mm}$ dorsal to the mPFC $(\mathrm{A} / \mathrm{P}+3.0, \mathrm{M} / \mathrm{L}-0.8, \mathrm{D} / \mathrm{V}$ $-3.5 \mathrm{~mm}$ from bregma) or OFC $(\mathrm{A} / \mathrm{P}+3.0, \mathrm{M} / \mathrm{L}+2.2, \mathrm{D} / \mathrm{V}$ $-3.5 \mathrm{~mm}$ from bregma). Extracellular glass microelectrodes were implanted vertically in the same coronal plane and, following a 20-min equilibration, baseline spontaneous local field potentials $(0.3-300 \mathrm{~Hz})$ were recorded for $5 \mathrm{~min}$. Chemical activation or disinhibition of the PFC was performed with NMDA $(0.75 \mu \mathrm{g}$ per $0.5 \mu \mathrm{l})$ or bicuculline $(0.1 \mu \mathrm{g}$ per $0.5 \mu \mathrm{l})$, respectively, infused through a 30 -gauge injection cannula protruding $2.0 \mathrm{~mm}$ past the end of the implanted guide cannula. LFPs were recorded for $60 \mathrm{~min}$ following drug administration. Power density was calculated in LabChart 7.1 (8K FFT, Hamming window, 0-60 Hz). Two counterbalanced infusions (one per region) were performed in each rat, with the second infusion occurring $>2 \mathrm{~h}$ after the first.

\section{Histology}

At the cessation of the experiments, the recording site was marked via electrophoretic ejection of Pontamine sky blue dye from the tip of the recording electrode $(-25 \mu \mathrm{A}$ constant current: 20-30 min) and iron deposited from the stimulating electrodes by passing constant current $(0.3 \mathrm{~mA}$, $10 \mathrm{~s})$ across the poles. Rats were decapitated and their brains removed, fixed for $24 \mathrm{~h}$ (3.7\% formaldehyde containing $1 \%$ potassium ferricyanide to enhance visualization of stimulating electrode tip location), and stored in $0.1 \mathrm{M}$ PBS until sectioning. Brains were cryoprotected $(10 \% \mathrm{w} / \mathrm{v}$ sucrose in PBS) until saturated and sectioned $(25 \mu \mathrm{m}$ coronal sections) on a cryostat (Shandon). Sections containing electrode placements were mounted onto gelatin-chrom alum-coated slides and stained with neutral red $(0.1 \%)$ and thionin acetate $(0.01 \%)$ for histochemical verification with reference to a stereotaxic atlas (Figure 1) (Paxinos and Watson, 1986).

\section{Analysis}

Electrophysiological analysis of dopamine neuron activity and cortical local field potentials were performed using commercially available computer software (LabChart v7.1). All data are represented as the mean \pm SEM unless otherwise specified. All statistics were calculated using the Prism software program (GraphPad). To examine the proportions of neurons excited or inhibited, an $\chi^{2}$ analysis was utilized that compared excited cells with those that were not excited (ie, no effect and inhibited) and inhibited neurons with those that were not inhibited (Table 1).

\section{Materials}

NMDA, 1(S),9(R)-(-)-Bicuculline methbromide, and chloral hydrate were all purchased from Sigma (St Louis, MO). All other chemicals and reagents were of either analytical or laboratory grade and were purchased from standard suppliers.

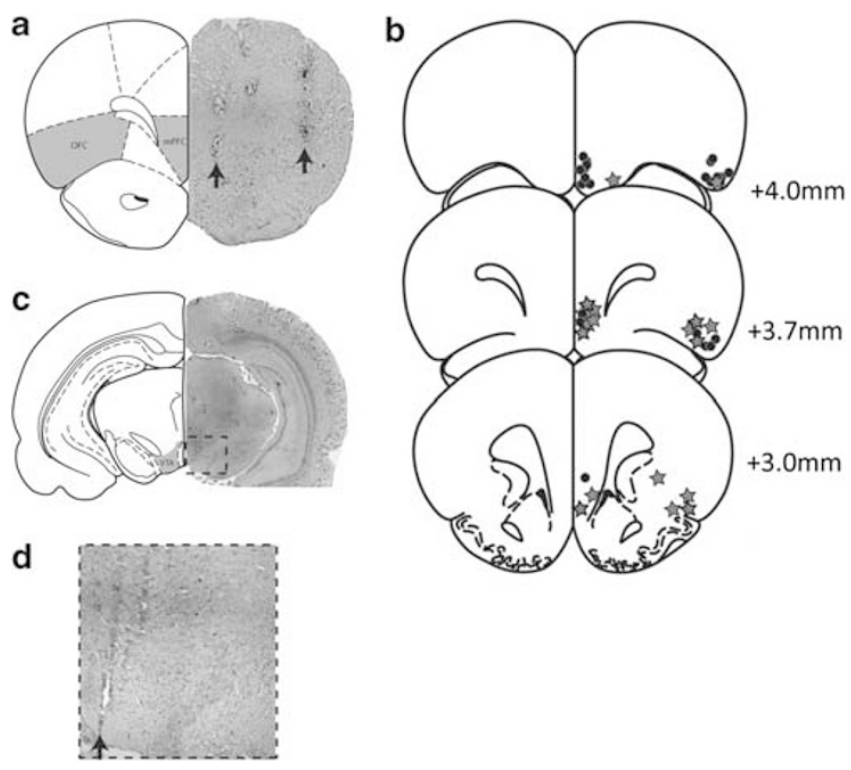

Figure I Representative brain section demonstrating the localization of stimulating electrode placements (arrows) within the mPFC and OFC (a). The locations of each recording electrode (star) or infusion site (circle) are depicted in (b), whereas a representative track throughout the VTA (arrow) is demonstrated in (c) and expanded in (d). The shaded areas illustrate electrode target sites-adapted from Paxinos and Watson (1986).

Table I PFC Stimulation Differentially Affects Dopamine Neuron Activity

\begin{tabular}{lccc}
\hline & No effect & Excitation & Inhibition \\
\hline mPFC, single pulse & $31 \%$ & $25 \%$ & $44 \%$ \\
mPFC, train & $2 \%$ & $94 \%^{\mathrm{a}}$ & $4 \%^{\mathrm{a}}$ \\
OFC, single pulse & $23 \%$ & $12 \%$ & $65 \%^{\mathrm{b}}$ \\
OFC, train & $10 \%$ & $40 \%^{\mathrm{a}, \mathrm{b}}$ & $50 \%^{\mathrm{b}}$ \\
\hline
\end{tabular}

a Significant difference between single-pulse and train stimulation.

bignificant difference between mPFC and OFC $\left(p<0.05 \chi^{2}, n=9\right.$ rats, $n=48$ neurons/group).

\section{RESULTS}

The PFC provides a significant input to dopamine neurons in the rat VTA (Gabbott et al, 2005; Sesack and Carr, 2002). Consistent with previous data (Aston-Jones et al, 2009; Overton et al, 1996; Tong et al, 1996, 1998), we report that single-pulse activation of the ventral mPFC induces either excitatory (in 25\% of dopamine neurons) or inhibitory (in $44 \%$ of dopamine neurons) effects (Figure 2 and Table 1 ). The variance of these responses is depicted in Supplementary Figures 1 and 2 . The excitatory effects ( $n=12$ neurons) were short latency $(19.6 \pm 4.5 \mathrm{~ms})$, in accord with previously reported antidromic latencies (Thierry et al, 1979), with a duration of $73 \pm 15 \mathrm{~ms}$, whereas the inhibition had a longer latency of $51.2 \pm 7.6 \mathrm{~ms}$ and a greater duration of $318 \pm 45 \mathrm{~ms} \quad(n=21$ neurons). In contrast, single-pulse activation of the OFC primarily inhibited spontaneous activity in the majority $(n=31$ neurons; $64.6 \%)$ of VTA 

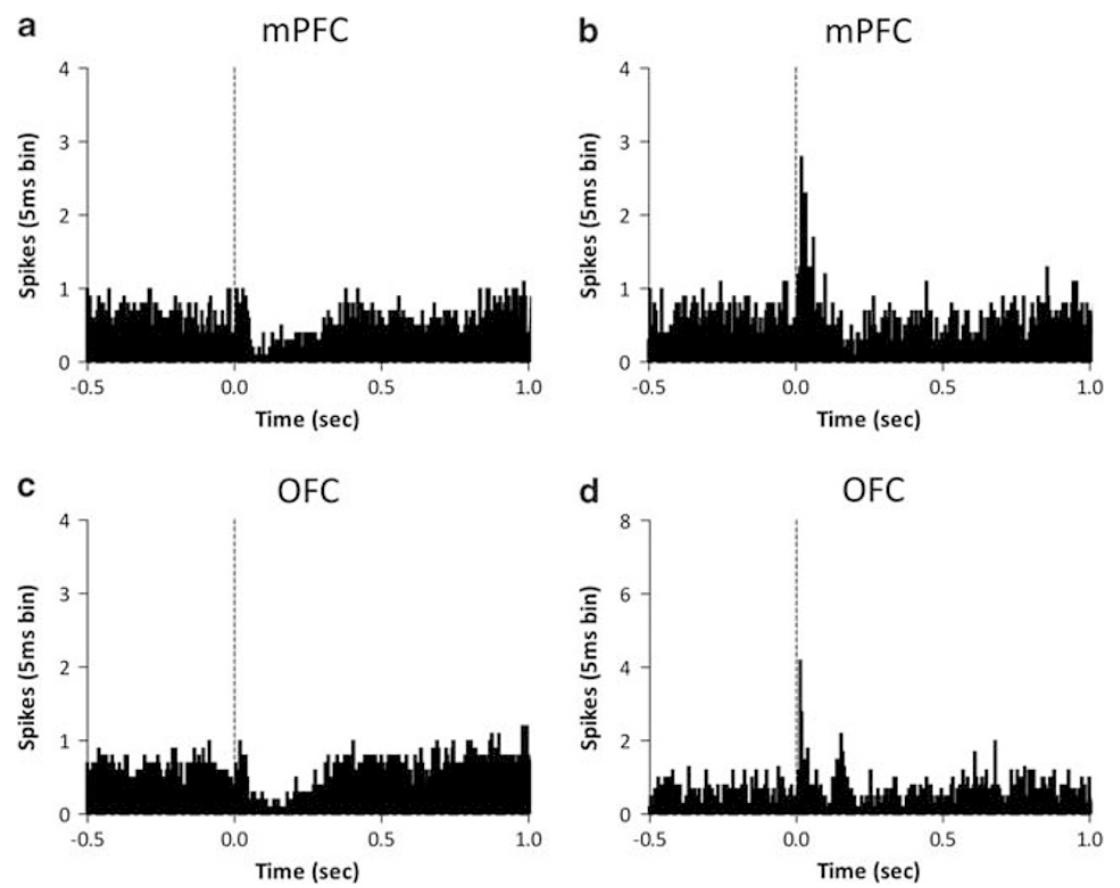

Figure 2 Single-pulse activation of the PFC differentially affects dopamine neuron activity. Peristimulus time histograms depict the average response of all recorded neurons demonstrating either inhibitory $(a, c)$ or excitatory $(b, d)$ effects of single-pulse electrical stimulation (vertical dashed line at time $=0$ ) of either the $\operatorname{mPFC}(a, b)$ or $\operatorname{OFC}(c, d)$. The proportion of neurons displaying each response is detailed in Table I $(n=9$ rats, $n=48$ neurons total).

dopamine neurons examined with a latency of $38.7 \pm 5.4 \mathrm{~ms}$ and a duration of $339 \pm 31 \mathrm{~ms}$ (Figure 2 and Table 1). Given that cortical neurons also fire higher-frequency bursts of activity (Burgos-Robles et al, 2007; Chang et al, 2010; Laviolette et al, 2005; Santini and Porter, 2010; Wang and Goldman-Rakic, 2004; Zhang and Shi, 1999), which are suggested to enhance the signaling of salient events and dampen interference from nonsalient events (Cooper, 2002; Lisman, 1997; Miller and Cohen, 2001), we also examined the response to burst-like stimulation of cortical afferents (four pulses, $65 \mathrm{~Hz}$ ). In contrast to that observed with single-pulse stimulation, burst-like activation of the mPFC resulted in a significant increase in spike firing in $93.8 \%$ of the neurons recorded (Figure 3 and Table 1). This increase in activity had a latency of $34.0 \pm 2.7 \mathrm{~ms}$ from the first stimulus in the train and a duration of $59 \pm 12 \mathrm{~ms}$. Moreover, this increase in spike firing was often $(64.4 \%$ of neurons) associated with a burst of action potentials by the dopamine neuron as determined by a maximum number of evoked spikes (in a $50-\mathrm{ms}$ bin) $>30$ (average $53.5 \pm 4.1$ spikes/bin; Figure 4). Interestingly, train stimulation of the OFC still produced an inhibitory response (latency $43.6 \pm 6.9 \mathrm{~ms}$; duration $468 \pm 54 \mathrm{~ms}$ ) in the majority of dopamine neurons ( $n=24$ neurons, 50\%), although an excitatory response was observed in $39.6 \%$ of neurons (c.f. $12.5 \%$ of neurons following single-pulse activation). It should be noted that repeated stimulus trains did not appear to produce enduring changes in dopamine neuron activity, as the average firing rate of all cells was consistent with that observed in untreated rats $(4.36 \pm 0.29 \mathrm{~Hz}, n=48$ neurons).

It is important to note that information gathered by recording individual dopamine neurons may not necessarily reflect the scope of the activity by the population of dopamine neurons in the VTA (Grace et al, 2007). To more comprehensively examine the effect of these independent PFC regions, NMDA was utilized to produce a sustained increase in cortical output, as reported previously (Floresco et al, 2003; Lodge and Grace, 2006a, b), while the activity population of dopamine neurons was examined. Rats that received control vehicle (Dulbecco's PBS) infusions into either the mPFC or OFC ( $n=5$ rats, 35 neurons) exhibited an average of $1.10 \pm 0.04$ spontaneously active dopamine neurons per electrode track that fired at an average rate of $4.6 \pm 0.3 \mathrm{~Hz}$ with $32 \pm 5 \%$ of action potentials occurring in bursts (Figure $5 \mathrm{a}-\mathrm{c}$ ), consistent with previous findings (Lodge and Grace, 2006a, b, 2007, 2008). Intra-OFC infusion of NMDA ( $n=5$ rats, 22 neurons) resulted in a significant decrease in dopamine neuron population activity $(0.65 \pm 0.09$ cells/track; $p<0.05$ one-way ANOVA; Figure 5a) without significantly affecting average burst firing $(21.4 \pm 4.7 \%)$ or firing rate $(4.7 \pm 0.4 \mathrm{~Hz})$ relative to control (Figure $2 \mathrm{~b}$ and $\mathrm{c}$ ). It should be noted that this is consistent with the number of neurons $(55 \%)$ that were completely inhibited following electrical activation of the OFC (defined as $>150 \mathrm{~ms}$ without activity following the stimulus). In contrast, intra-mPFC infusions of NMDA ( $n=6$ rats, 44 neurons) did not significantly alter dopamine neuron population activity $(1.22 \pm 0.12$ cells/track; Figure $5 \mathrm{a})$, average burst firing $(31.8 \pm 4.2 \%)$ or firing rate $(4.48 \pm 0.3 \mathrm{~Hz})$ relative to control (Figure $2 \mathrm{~b}$ and c). Based on the effects of single-pulse electrical activation of the mPFC, it is likely that NMDA activation increases and decreases distinct sub-populations of dopamine neurons with the net result being no observable difference in the average activity of the population of neurons examined. 

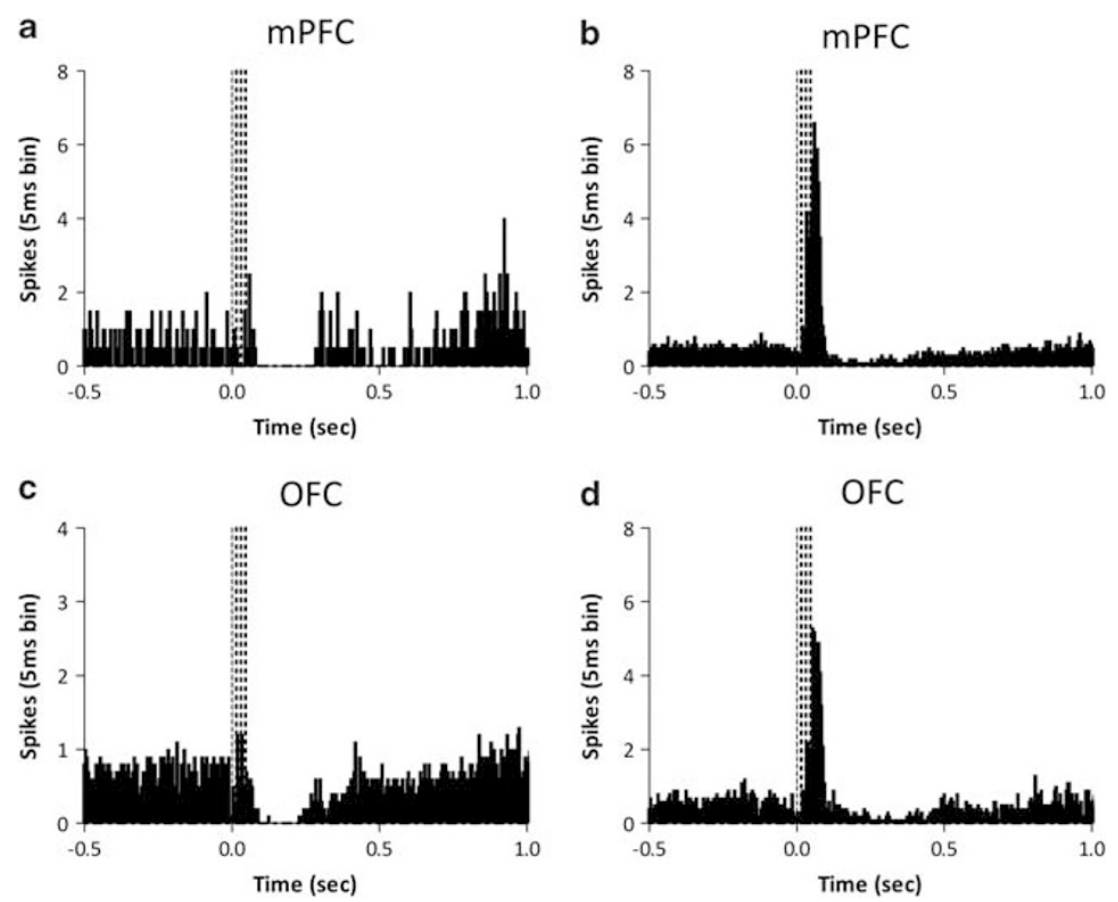

Figure 3 Burst-like pulse activation of the PFC dramatically alters dopamine neuron responses. Peristimulus time histograms depict the average response of all recorded neurons demonstrating either inhibitory $(a, c)$ or excitatory (b, d) effects of electrical stimulus trains (four pulses at $65 \mathrm{~Hz}$ : vertical dashed lines) of either the $\operatorname{mPFC}(a, b)$ or OFC $(c, d)$. The proportion of neurons displaying each response is detailed in Table I $(n=9$ rats, $n=48$ neurons total).
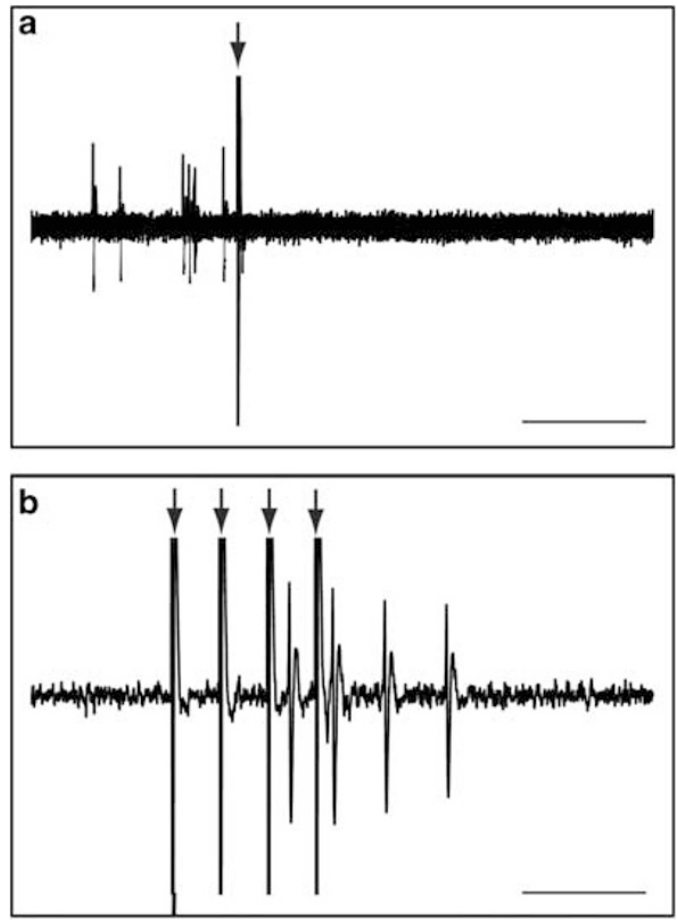

Figure 4 Representative extracellular voltage traces demonstrating a dopamine neuron response to mPFC stimulation. The trace in (a) depicts the inhibition of dopamine neuron firing by single-pulse mPFC activation. Interestingly, the same neuron exhibited a strong burst of excitation in response to mPFC train stimulation (b). The arrows depict stimulus artifacts. Scale bar represents $100 \mathrm{~ms}$ in (a) and $40 \mathrm{~ms}$ in (b).
To examine the effect of cortical burst firing on the population of dopamine neurons in the VTA, we investigated the ability of the $\mathrm{GABA}_{\mathrm{A}}$ receptor antagonist, bicuculline, to disinhibit cortical neurons. The local administration of bicuculline induced high-amplitude field potentials in both the mPFC and OFC that lasted for $\sim 30$ min (Figure 6). These spike-wave discharges have been demonstrated to correspond with coordinated bursting of pyramidal neurons (Steriade et al, 1998). Intra-OFC infusion of bicuculline ( $n=6$ rats, 41 neurons) resulted in a significantly greater dopamine neuron population activity during the first $30 \mathrm{~min}(1.50 \pm 0.18$ cells/track c.f. $0.90 \pm 0.11$ cells/track; $p<0.05 t$-test; Figure $7 \mathrm{a})$ without significant differences in average burst firing $(29 \pm 5 \%$ c.f. $29 \pm 7 \%$; Figure $7 \mathrm{c})$ or firing rate $(4.4 \pm 0.5 \mathrm{~Hz}$ c.f. $4.5 \pm 0.4 \mathrm{~Hz}$; Figure $7 \mathrm{~b}$ ). In contrast, intra-mPFC infusion of bicuculline ( $n=6$ rats, 40 neurons) resulted in significantly higher burst firing during the first $30 \mathrm{~min}(40.7 \pm 6 \%$ c.f. $17.5 \pm 5 \%$; $p<0.05 t$-test; Figure $7 \mathrm{c}$ ); a trend for a greater population activity $(1.56 \pm 0.19$ cells/track c.f. $1.06 \pm 0.06$ cells/track; $p=0.07 t$-test; Figure 7a) while having no significant effect on average firing rate $(4.5 \pm 0.5 \mathrm{~Hz}$ c.f. $3.8 \pm 0.5 \mathrm{~Hz}$; Figure 7b). Thus, these data appear to parallel those observed following train stimulation of the PFC, ie, an enhanced dopamine neuron response to $\mathrm{mPFC}$ activation. It should be noted that dopamine neuron activity observed $>30$ min following bicuculline administration was not significantly different to that observed following vehicle administration (all $p>0.05$ one-way ANOVA). For this reason, a within-subject comparison was utilized when 

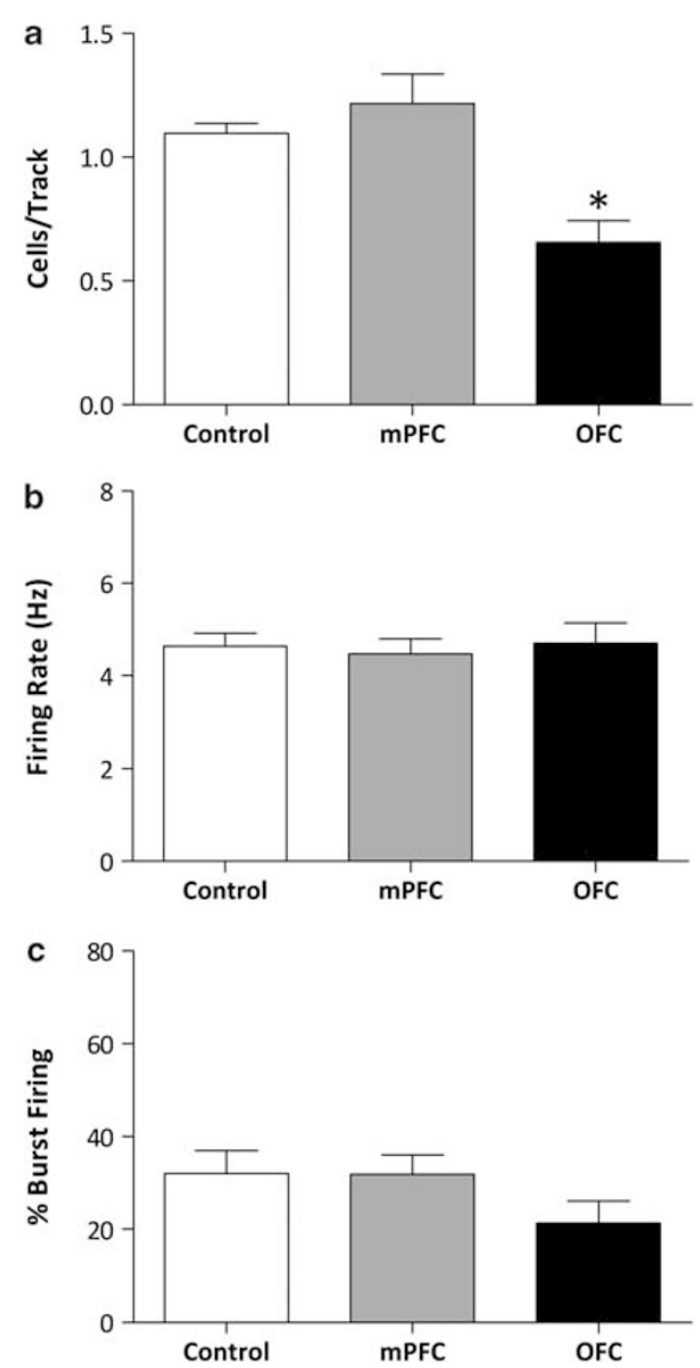

Figure 5 NMDA activation of the OFC decreases dopamine system function. Three parameters of activity were recorded: population activity (number of spontaneously firing dopamine neurons per electrode track (a)), average firing rate (b), and average percent spikes fired in bursts (c). Consistent with the effects of electrical stimulation, NMDA activation of the OFC significantly reduced dopamine neuron population activity. In contrast, NMDA activation of the mPFC produced no observable effects on the population of dopamine neurons in the VTA. Based on the effects of single-pulse electrical activation of the mPFC, we posit that NMDA activation increases and decreases distinct sub-populations of dopamine neurons with the net result being no observable difference in the average activity of the population of neurons examined. ${ }^{*} p<0.05$ one-way ANOVA-Dunnett's post hoc: $n=5$ to 6 rats/group, $n=22-44$ neurons/group.

examining the effects of bicuculline on dopamine neuron activity.

\section{DISCUSSION}

In this study we demonstrate that the mPFC and OFC differentially regulate dopamine neuron activity and, moreover, the pattern of cortical activation is critical for determining dopamine system output.

It has been known for some time that the MPFC can regulate dopamine neuron activity and that this regulation

likely reflects the pattern of cortical innervation of the VTA (Aston-Jones et al, 2009; Overton et al, 1996; Tong et al, 1996, 1998). Indeed, single-pulse or NMDA activation of the mPFC activated or inhibited distinct sub-populations of VTA dopamine neurons. Based on anatomical studies by Carr and Sesack (2000), it is likely that the excited cells are mesocortical neurons receiving monosynaptic inputs from glutamatergic mPFC neurons; indeed, this is supported by the short latency $(19.6 \pm 4.5 \mathrm{~ms})$ of the evoked response. In contrast, dopamine neurons inhibited by the $\mathrm{mPFC}$ had a long latency $(51.2 \pm 7.6 \mathrm{~ms})$ and are likely mesolimbic neurons, innervated by recurrent collaterals from GABAergic neurons (Carr and Sesack, 2000). Thus, tonic single-spike activity in the mPFC is postulated to regulate the balance between mesolimbic and mesocortical transmission.

It is well established that pyramidal neurons of the cortex display transient elevations in activity in response to cognitive tasks (Homayoun and Moghaddam, 2006a; Jackson et al, 2004; Jung et al, 1998). Furthermore, a subset of pyramidal neurons in the $\mathrm{mPFC}$, specifically those layer 5 neurons known to innervate the VTA (Gabbott et al, 2005), encode information as high-frequency bursts (Burgos-Robles et al, 2007; Chang et al, 2010; Laviolette et al, 2005; Santini and Porter, 2010; Wang and Goldman-Rakic, 2004; Zhang and Shi, 1999). Thus, mPFC neurons can display periods of intense burst firing in response to salient stimuli (Burgos-Robles et al, 2007; Chang et al, 2010; Laviolette et al, 2005; Santini and Porter, 2010; Wang and Goldman-Rakic, 2004; Zhang and Shi, 1999); however, the relevance of this distinct firing mode, in the context of dopamine neuron activity, has not been examined. In this study we demonstrate that burst-like activation of the mPFC dramatically augments dopamine neuron activity. Specifically, in contrast to the relatively discrete effects of singlepulse activation, burst-like stimulation of the mPFC produced a massive increase in dopamine neuron activity in the vast majority of neurons recorded. This is consistent with previous studies examining dopamine efflux in the nucleus accumbens in response to $\mathrm{mPFC}$ activation. Specifically, low-frequency $(10 \mathrm{~Hz})$ electrical stimulation of the $\mathrm{mPFC}$ has been demonstrated to decrease accumbens dopamine levels, consistent with a decreased activity of putative mesolimbic neurons, whereas higher-frequency activation $(60 \mathrm{~Hz})$ of the mPFC produces the opposite response, ie, an increase in dopamine efflux (Jackson et al, 2001). Thus, although single-spike activity in the mPFC likely sets the balance between mesolimbic and mesocortical activity, salient events that promote burst firing of the mPFC are likely to induce a more substantial dopamine signal that promotes attention to the stimulus. Interestingly, given the relatively sparse innervation of the VTA by the dorsolateral PFC in primates (Frankle et al, 2006), this is likely a prominent form of communication between PFC and dopamine systems.

It should be noted that train stimulation of the mPFC induced a burst of action potentials by the dopamine neuron, even in neurons inhibited by single-pulse stimulation. At first, this may seem at odds with the relatively discrete innervation of the VTA by the mPFC (Carr and Sesack, 2000); however, there are a number of ways by which train stimulation can induce effects distinct to that 

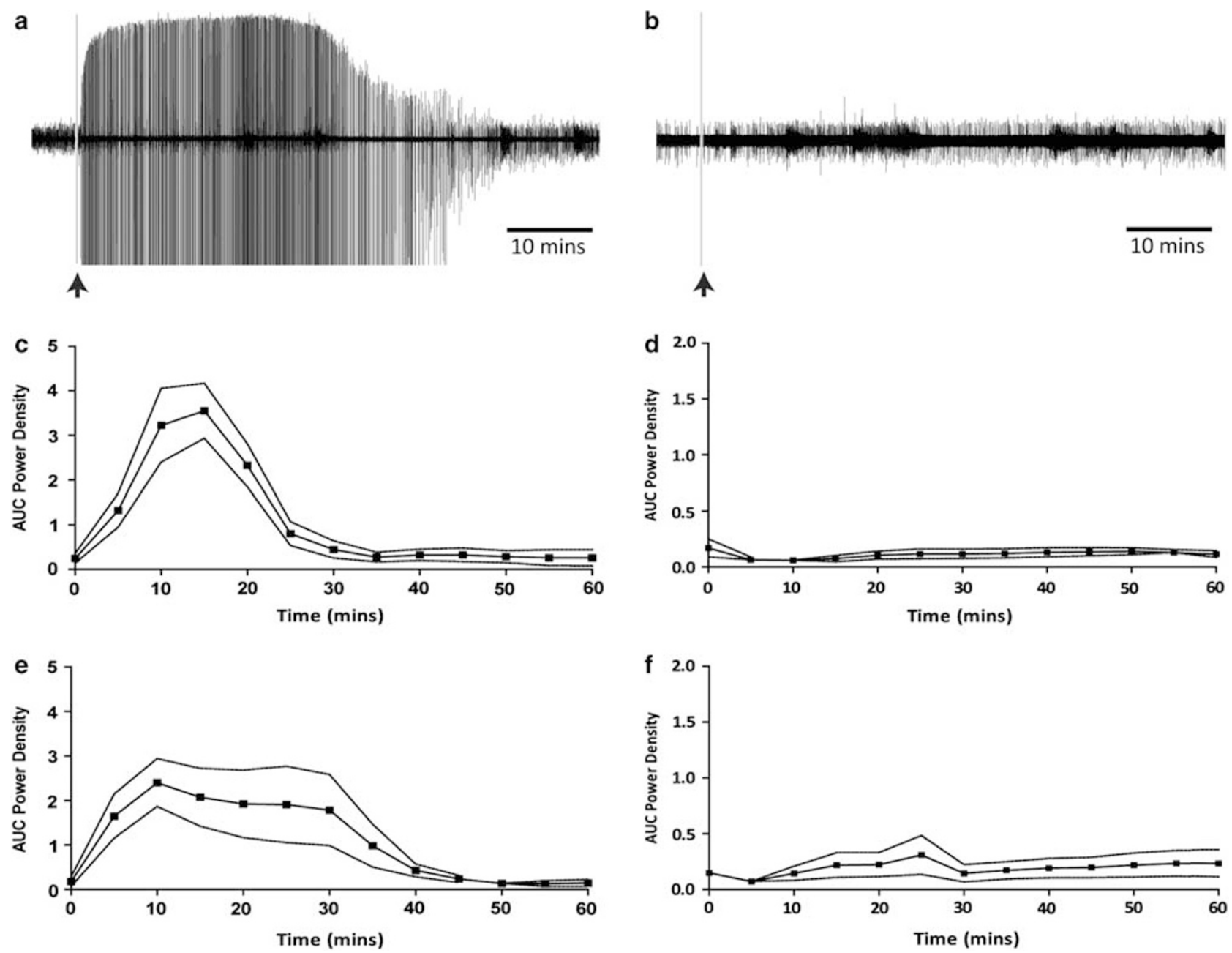

Figure 6 Phasic activation of the PFC by bicuculline. Intra-PFC infusions of bicuculline $(0.1 \mu \mathrm{g}$ per $0.5 \mu \mathrm{l})$, but not NMDA $(0.75 \mu \mathrm{g}$ per $0.5 \mu \mathrm{l})$, induced spike-wave discharges that persisted for $\sim 30 \mathrm{~min}$. Raw voltage traces are depicted from a representative experiment with either bicuculline (a) or NMDA (b). Group data depict the mean \pm SEM of the area under the curve (AUC) of the power spectrum (8K FFT, Hamming window, 0-60 Hz) for bicuculline or NMDA infusions into the $\mathrm{mPFC}(\mathrm{c}, \mathrm{d}$ respectively) and OFC ( $\mathrm{e}, \mathrm{f}$ respectively). $n=4$ rats/group.

observed with single pulse. One possibility is through the liberation of peptide modulators such as cholecystokinin, known to promote burst firing of VTA dopamine neurons (Skirboll et al, 1981). Another potential explanation is that the increase in dopamine neuron activity may not necessarily be synaptic in origin. Thus, microdialysis studies have demonstrated that although low-frequency $(<16 \mathrm{~Hz})$ activation of the mPFC does not significantly alter glutamate levels within the VTA, stimulation at higher frequencies $(>64 \mathrm{~Hz})$ induces a $\sim 300 \%$ increase in extrasynaptic glutamate (Rossetti et al, 1998). Such an increase in extracellular glutamate could seemingly affect dopamine neurons that do not receive a direct synaptic input from the mPFC. Given that microiontophoretically applied glutamate not only induces burst firing in dopamine neurons (Lodge and Grace, 2006b), but also activates 'silent' neurons in the VTA (Grace and Bunney, 1984b), it is likely that phasic $\mathrm{mPFC}$ activity produces profound effects on the population of dopamine neurons in the VTA. Indeed, in this study we demonstrate that intra-mPFC bicuculline administration induces an increase in both dopamine neuron population activity and burst firing. Taken together, it appears that distinct activity states of the mPFC differentially regulate dopamine system function. More specifically, tonic MPFC activity likely regulates the balance between mesocortical and mesolimbic transmission, whereas salient stimuli that induce burst firing of layer 5 pyramidal neurons are likely to cause a massive increase in dopamine neuron activity, by increasing burst firing as well as increasing the number of spontaneously active neurons that convey that signal.

One mediator that has been demonstrated to induce burst firing of layer 5 pyramidal cells in the mPFC is dopamine itself, via an action on D2-like receptors (Wang and Goldman-Rakic, 2004). Therefore, this provides a potential positive feedback loop by which salient events promote burst firing of pyramidal neurons that then induce phasic activity in dopamine neurons that, in turn, augments burst firing of mPFC neurons. Thus, it is likely that complementary regions act as a brake in order to stop this positive feedback cycle, with one potential region being the OFC. In contrast to the significant literature examining the function of the mPFC, a role for the OFC in the regulation of dopamine system function has not yet been demonstrated. 

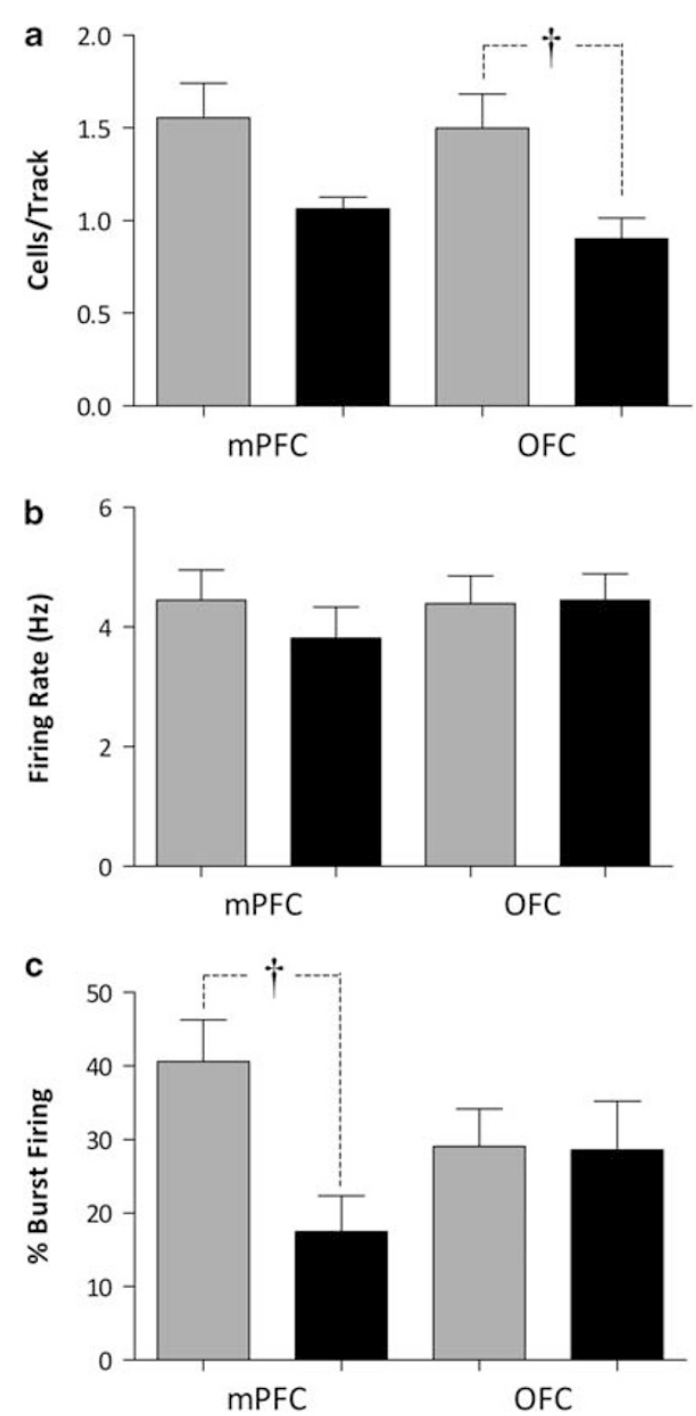

Figure 7 Bicuculline disinhibition of the PFC mimics burst-like stimulation. Three parameters of activity were recorded: population activity (number of spontaneously firing dopamine neurons per electrode track (a)), average firing rate (b), and average percent spikes fired in bursts (c). Because of the transient effects of bicuculline (see Figure 6), data are presented as the first $30 \mathrm{~min}$ following drug infusion (gray bars) compared with data collected after (black bars). ${ }^{\dagger} p<0.05$ t-test: $n=6$ rats/group, $n=21-23$ neurons/group.

Based on anatomical studies, it is likely that the OFC can modulate VTA activity either directly or indirectly (Gabbott et al, 2005). Indeed, we now demonstrate that the OFC can regulate the activity of the majority of dopamine neurons in the VTA. Specifically, single-pulse activation of the OFC potently inhibits spontaneous activity in a significant proportion of the dopamine neurons examined. Furthermore, although burst-like stimulation of the OFC still resulted in an inhibitory effect in $50 \%$ of the VTA dopamine neurons, a phasic activation was observed in a subset (40\%) of neurons. Thus, it appears that the OFC regulation of dopamine neuron activity is secondary to activation of GABAergic neurons that likely originate within the accumbens or VTA itself. As mentioned above, how the OFC regulates dopamine system function is of critical importance given the role of the OFC in adaptive responding

(Schoenbaum et al, 2009; Takahashi et al, 2009). Thus, it has been demonstrated that the OFC is a critical region associated with response inhibition. Specifically, recent electrophysiological studies have demonstrated that when reward signaling of OFC neurons is increased, prediction errors observed in dopamine neurons are attenuated (Takahashi et al, 2009). These data suggest that the OFC may negatively regulate dopamine neuron activity. In this study we demonstrate that OFC activation does indeed result in a strong inhibition of spontaneous activity within the VTA, and may therefore provide the foundation by which the OFC can signal learning when outcomes are not as expected, ie, during overexpectation (Schoenbaum et al, 2009; Takahashi et al, 2009).

Taken as a whole, these data demonstrate that the mPFC and OFC differentially regulate dopamine neuron activity, and that the pattern of cortical activation is critical for determining dopamine system output. These data further suggest that modulation of different regions of the frontal cortex, which themselves have been implicated in different aspects of cognition (ie, executive function and error prediction), produces functionally divergent effects on the dopamine system. Given that these regions often display discordant responses, such as that observed following repeated psychostimulant administration (Homayoun and Moghaddam, 2006b), understanding how these PFC regions modulate dopamine system function is of critical relevance to a number of neuropsychiatric disorders including schizophrenia, ADHD, and addiction.

\section{ACKNOWLEDGEMENTS}

We thank Amber Asher for her valuable technical assistance. This work was supported by the USPHS MH090067, the Department of Pharmacology, UTHSCSA, and a NARSAD award from the Maltz Family Foundation.

\section{DISCLOSURE}

The author declares no conflict of interest.

\section{REFERENCES}

Angel A, Gratton DA (1982). The effect of anaesthetic agents on cerebral cortical responses in the rat. Br J Pharmacol 76: 541-549.

Aston-Jones G, Bloom FE (1981). Norepinephrine-containing locus coeruleus neurons in behaving rats exhibit pronounced responses to non-noxious environmental stimuli. J Neurosci 1: 887-900.

Aston-Jones G, Smith RJ, Moorman DE, Richardson KA (2009). Role of lateral hypothalamic orexin neurons in reward processing and addiction. Neuropharmacology 56(Suppl 1): 112-121.

Burgos-Robles A, Vidal-Gonzalez I, Santini E, Quirk GJ (2007). Consolidation of fear extinction requires NMDA receptordependent bursting in the ventromedial prefrontal cortex. Neuron 53: 871-880.

Carr DB, Sesack SR (2000). Projections from the rat prefrontal cortex to the ventral tegmental area: target specificity in the synaptic associations with mesoaccumbens and mesocortical neurons. J Neurosci 20: 3864-3873.

Chagnac-Amitai Y, Luhmann HJ, Prince DA (1990). Burst generating and regular spiking layer 5 pyramidal neurons of 
rat neocortex have different morphological features. J Comp Neurol 296: 598-613.

Chang CH, Berke JD, Maren S (2010). Single-unit activity in the medial prefrontal cortex during immediate and delayed extinction of fear in rats. PLOS ONE 5: e11971.

Clayton EC, Rajkowski J, Cohen JD, Aston-Jones G (2004). Phasic activation of monkey locus ceruleus neurons by simple decisions in a forced-choice task. J Neurosci 24: 9914-9920.

Cooper DC (2002). The significance of action potential bursting in the brain reward circuit. Neurochem Int 41: 333-340.

Floresco SB, Magyar O, Ghods-Sharifi S, Vexelman C, Tse MTL (2006). Multiple dopamine receptor subtypes in the medial prefrontal cortex of the rat regulate set-shifting. Neuropsychopharmacology 31: 297-309.

Floresco SB, West AR, Ash B, Moore H, Grace AA (2003). Afferent modulation of dopamine neuron firing differentially regulates tonic and phasic dopamine transmission. Nat Neurosci 6: 968-973.

Frankle WG, Laruelle M, Haber SN (2006). Prefrontal cortical projections to the midbrain in primates: evidence for a sparse connection. Neuropsychopharmacology 31: 1627-1636.

Gabbott PLA, Warner TA, Jays PRL, Salway P, Busby SJ (2005). Prefrontal cortex in the rat: projections to subcortical autonomic, motor, and limbic centers. J Comp Neurol 492: 145-177.

Grace AA, Bunney BS (1983). Intracellular and extracellular electrophysiology of nigral dopaminergic neurons-1. Identification and characterization. Neuroscience 10: 301-315.

Grace AA, Bunney BS (1984a). The control of firing pattern in nigral dopamine neurons: burst firing. J Neurosci 4: 2877-2890.

Grace AA, Bunney BS (1984b). The control of firing pattern in nigral dopamine neurons: single spike firing. J Neurosci 4: 2866-2876.

Grace AA, Floresco SB, Goto Y, Lodge DJ (2007). Regulation of firing of dopaminergic neurons and control of goal-directed behaviors. Trends Neurosci 30: 220-227.

Homayoun H, Moghaddam B (2006a). Bursting of prefrontal cortex neurons in awake rats is regulated by metabotropic glutamate 5 (mGlu5) receptors: rate-dependent influence and interaction with NMDA receptors. Cereb Cortex 16: 93-105.

Homayoun H, Moghaddam B (2006b). Progression of cellular adaptations in medial prefrontal and orbitofrontal cortex in response to repeated amphetamine. J Neurosci 26: 8025-8039.

Hyland BI, Reynolds JNJ, Hay J, Perk CG, Miller R (2002). Firing modes of midbrain dopamine cells in the freely moving rat. Neuroscience 114: 475-492.

Jackson ME, Frost AS, Moghaddam B (2001). Stimulation of prefrontal cortex at physiologically relevant frequencies inhibits dopamine release in the nucleus accumbens. J Neurochem 78: 920-923.

Jackson ME, Homayoun H, Moghaddam B (2004). NMDA receptor hypofunction produces concomitant firing rate potentiation burst activity reduction in the prefrontal cortex. Proc Natl Acad Sci USA 101: 8467-8472.

Jung MW, Qin Y, McNaughton BL, Barnes CA (1998). Firing characteristics of deep layer neurons in prefrontal cortex in rats performing spatial working memory tasks. Cereb Cortex 8: 437-450.

Laviolette SR, Lipski WJ, Grace AA (2005). A subpopulation of neurons in the medial prefrontal cortex encodes emotional learning with burst and frequency codes through a dopamine D4 receptor-dependent basolateral amygdala input. J Neurosci 25: 6066-6075.

Lisman JE (1997). Bursts as a unit of neural information: making unreliable synapses reliable. Trends Neurosci 20: 38-43.

Lodge DJ, Grace AA (2006a). The hippocampus modulates dopamine neuron responsivity by regulating the intensity of phasic neuron activation. Neuropsychopharmacology 31: $1356-1361$.
Lodge DJ, Grace AA (2006b). The laterodorsal tegmentum is essential for burst firing of ventral tegmental area dopamine neurons. Proc Natl Acad Sci USA 103: 5167-5172.

Lodge DJ, Grace AA (2007). Aberrant hippocampal activity underlies the dopamine dysregulation in an animal model of schizophrenia. J Neurosci 27: 11424-11430.

Lodge DJ, Grace AA (2008). Amphetamine activation of hippocampal drive of mesolimbic dopamine neurons: a mechanism of behavioral sensitization. J Neurosci 28: 7876-7882.

Margolis EB, Lock H, Hjelmstad GO, Fields HL (2006). The ventral tegmental area revisited: is there an electrophysiological marker for dopaminergic neurons? J Physiol 577: 907-924.

McAlonan K, Brown VJ (2003). Orbital prefrontal cortex mediates reversal learning and not attentional set shifting in the rat. Behav Brain Res 146: 97-103.

Miller EK, Cohen JD (2001). An integrative theory of prefrontal cortex function. Annu Rev Neurosci 24: 167-202.

Nishiike S, Takeda N, Kubo T, Nakamura S (1997). Neurons in rostral ventrolateral medulla mediate vestibular inhibition of locus coeruleus in rats. Neuroscience 77: 219-232.

O'Doherty J, Critchley H, Deichmann R, Dolan RJ (2003). Dissociating valence of outcome from behavioral control in human orbital and ventral prefrontal cortices. J Neurosci 23: 7931-7939.

Overton PG, Tong ZY, Clark D (1996). A pharmacological analysis of the burst events induced in midbrain dopaminergic neurons by electrical stimulation of the prefrontal cortex in the rat. J Neural Transm 103: 523-540.

Paxinos G, Watson C (1986). The Rat Brain in Stereotaxic Coordinates 2nd edn Academic Press: Sydney, Australia.

Rossetti ZL, Marcangione C, Wise RA (1998). Increase of extracellular glutamate and expression of Fos-like immunoreactivity in the ventral tegmental area in response to electrical stimulation of the prefrontal cortex. J Neurochem 70: 1503-1512.

Santini E, Porter JT (2010). M-type potassium channels modulate the intrinsic excitability of infralimbic neurons and regulate fear expression and extinction. J Neurosci 30: 12379-12386.

Schoenbaum G, Roesch MR, Stalnaker TA, Takahashi YK (2009). A new perspective on the role of the orbitofrontal cortex in adaptive behaviour. Nat Rev Neurosci 10: 885-892.

Sesack SR, Carr DB (2002). Selective prefrontal cortex inputs to dopamine cells: implications for schizophrenia. Physiol Behav 77: 513-517.

Skirboll LR, Grace AA, Hommer DW (1981). Peptide-monoamine coexistence: studies of the actions of cholecystokinin-like peptide on the electrical activity of midbrain dopamine neurons. Neuroscience 6: 2111-2124.

Steinfels GF, Heym J, Strecker RE, Jacobs BL (1983). Behavioral correlates of dopaminergic unit activity in freely moving cats. Brain Res 258: 217-228.

Steriade M, Amzica F, Neckelmann D, Timofeev I (1998). Spikewave complexes and fast components of cortically generated seizures. II. Extra- and intracellular patterns. J Neurophysiol 80: 1456-1479.

Takahashi YK, Roesch MR, Stalnaker TA, Haney RZ, Calu DJ, Taylor AR et al (2009). The orbitofrontal cortex and ventral tegmental area are necessary for learning from unexpected outcomes. Neuron 62: 269-280.

Thierry AM, Deniau JM, Feger J (1979). Effects of stimulation of the frontal cortex on identified output VMT cells in the rat. Neurosci Lett 15: 103-107.

Tong ZY, Overton PG, Clark D (1996). Stimulation of the prefrontal cortex in the rat induces patterns of activity in midbrain dopaminergic neurons which resemble natural burst events. Synapse 22: 195-208.

Tong ZY, Overton PG, Martinez-Cué C, Clark D (1998). Do nondopaminergic neurons in the ventral tegmental area play a role in the responses elicited in A10 dopaminergic neurons by 


\begin{tabular}{rr} 
npg & PFC control of dopamine neuron activity \\
\hline 1236 & DJ Lodge
\end{tabular}

electrical stimulation of the prefrontal cortex? Exp Brain Res 118: 466-476.

Ungless MA, Magill PJ, Bolam JP (2004). Uniform inhibition of dopamine neurons in the ventral tegmental area by aversive stimuli. Science 303: 2040-2042.

Wang Y, Goldman-Rakic PS (2004). D2 receptor regulation of synaptic burst firing in prefrontal cortical pyramidal neurons. Proc Natl Acad Sci USA 101: 5093-5098.
Whim MD, Lloyd PE (1989). Frequency-dependent release of peptide cotransmitters from identified cholinergic motor neurons in Aplysia. Proc Natl Acad Sci USA 86: 9034-9038.

Zhang XX, Shi WX (1999). Dendritic glutamate-induced bursting in prefrontal pyramidal cells: role of NMDA and non-NMDA receptors. Acta Pharmacol Sin 20: $1125-1131$

Supplementary Information accompanies the paper on the Neuropsychopharmacology website (http://www.nature.com/npp) 